九州大学学術情報リポジトリ

Kyushu University Institutional Repository

\title{
INVERSE PARTITION PROBLEMS
}

Iwamoto, Seiichi

Department of Economic Engineering, Faculty of Economics, Kyushu University

Ueno, Takayuki

Department of Economic Engineering, Faculty of Economics, Kyushu University

https://doi . org/10.5109/13481

出版情報: Bulletin of informatics and cybernetics. 31 (1), pp.67-90, 1999-03. Research Association of Statistical Sciences バージョン :

権利関係 : 


\title{
INVERSE PARTITION PROBLEMS
}

\author{
By
}

Seiichi Iwamoto* and Takayuki UEno*

\begin{abstract}
We analyze a partition problem and its inverse problem both in discrete variables and in two continuous ones through dynamic programming. We show that an inverse relation and an envelopping relation hold in each case. It is shown that one optimal solution in discrete partition is expressed by the other through either upper-semi inverse function or lower-semi inverse function and that optimal solutions in continuous partition through the (regular) inverse function. As a result, we show that the optimal partition is to partition equally in essence any quantity into the quantities of the same size of $e$. We call this optimal policy Euler partition rule.
\end{abstract}

\section{Introduction}

The partition problem is one of traditional mathematical optimization problems. It is also called division problem in Beckmann and Lademan(1956), Golmb(1968,80) and others or allocation problem in Beckmann(1968), Ibaraki and Katoh(1988), and others. In this paper it is to partition any given nonnegative quantity into any number of nonnegative ones to maximize the product (multiplicated quantity). In the paper we consider three partitions; a discrete partition and two continuous partitions. We propose an inversion of each (main) partition problem from the view point of dynamic programming (Bellman(1975), Iwamoto(1987), Sniedovich(1992)). We show that an inverse relation holds between main and inverse partition problems. Further, by relating the partition problem with the family of fixed partition problems, we derive an envelopping relation between the partition problem and the family.

In section 2, we consider the discrete partition. We analyze both main and inverse partition problems through dynamic programming (Iwamoto(1977a,1977b,1977c)). We propose upper-semi inverse function and lower-semi inverse function. It is shown that one optimal solution is characterized by the other through either inverse function (inverse theorem).

In section 3 , we consider the partition of any nonnegative real into finitely many ones. We state the inverse theorem through the (regular) inverse function.

\footnotetext{
* Department of Economic Engineering, Faculty of Economics, Kyushu University 27, Fukuoka 8128581 , Japan

e-mail: iwamoto\{ueno\}@en.kyushu-u.ac.jp
} 
In section4, we are concerned with partition into continuously many ones. We derive the inverse relation by Bellman equation in continuous dynamic programming (Iwamoto and Wang $(1983,1986)$ ).

Throughout the analysis of three main partition problems, we show that the Euler partition rule is an optimal partition. That is, the optimal partition is to partition equally in essence any quantity into the quantities of the same size of the Euler number $e$.

\section{Discrete Partition}

In this section, we consider a pair of partition problems of any natural number $n$ into natural numbers.

\subsection{Regular Partition Problems}

In this subsection, we are concerned with partition into any number of natural numbers. We consider the main partition problem $(\operatorname{Golmb}(1968,1980))$ :

$$
\begin{array}{lll}
\text { Maximize } & n_{1} n_{2} \cdots n_{t} \\
\operatorname{MPP}(n) & \text { subject to } & \text { (i) } n_{1}+n_{2}+\cdots+n_{t}=n \\
& \text { (ii) } n_{1}, n_{2}, \ldots, n_{t}, t \in N
\end{array}
$$

and its inverse partition problem:

$$
\begin{array}{lll}
\text { minimize } & n_{1}+n_{2}+\cdots+n_{t} \\
\operatorname{IPP}(n) \quad \text { subject to } & \text { (i) } n_{1} n_{2} \cdots n_{t} \geq n \\
& \text { (ii) } n_{1}, n_{2}, \ldots, n_{t}, t \in N
\end{array}
$$

where $n \in N$.

Let $f(n), g(n)$ be the maximum value and the minimum value, respectively. First we have the monotonicity of optimum value functions $f(\cdot), g(\cdot)$ as follows:

LEMMA 2.1. The maximum value function $f: N \rightarrow N$ is strictly increasing but the minimum value function $g: N \rightarrow N$ is nondecreasing. Both go to $\infty$ as so does $n$.

Second we have the recursive equations:

ThEOREM 2.2 .

$$
\begin{array}{ll}
f(n) & =\operatorname{Max}_{1 \leq k<n}[k f(n-k)] \quad n \geq 5 \\
f(n) & =n \quad n=1,2,3,4 \\
g(n) & =\min _{\substack{1<k, l<n \\
k l \geq n}}[k+g(l)] \quad n \geq 6 \\
g(n) & =n \quad n=1,2,3,4,5 .
\end{array}
$$


We note that the minimization in (5) is taken over the set of all pairs $(k, l)$ satisfying the condition $1<k, l<n, k l \geq n$.

Let $\pi^{*}(n)$ be the the maximizer in (3) and $\hat{\sigma}(n)$ be the value of $k$ for the minimizer in $(5)$, respectively.

\subsection{Inverse Theorems}

Furthermore, we have the inverse relationship between Main and Inverse Partition Problems:

Theorem 2.3. (Weak Inverse Theorem)

(i) $\quad g(f(n))=n, \quad f(g(n)) \geq n \quad n \in N$

(ii) $\quad f(g(n))=n \quad n=3^{m}, 4 \cdot 3^{m-1}, 2 \cdot 3^{m}$ for $m \in N$.

Proof (i) Let $n \in N$. First we have a maximum solution:

$$
f(n)=n_{1} n_{2} \cdots n_{t}
$$

for some $n_{1}, n_{2}, \ldots, n_{t}, t \in N$ satisfying

$$
n_{1}+n_{2}+\cdots+n_{t}=n .
$$

Thus we have

$$
g(f(n)) \leq n .
$$

On the other hand, set $g(f(n))<n$. Then there exist $m_{1}, m_{2}, \ldots, m_{s}, s \in N$ such that

$$
g(f(n))=m_{1}+m_{2}+\cdots+m_{s}<n, \quad m_{1} m_{2} \cdots m_{s} \geq f(n) .
$$

Therefore we can choose $m_{1}^{*}>m_{1}$ satisfying

$$
m_{1}^{*}+m_{2}+\cdots+m_{s}=n, \quad m_{1}^{*} m_{2} \cdots m_{s}>m_{1} m_{2} \cdots m_{s} \geq f(n) .
$$

This contradicts the maximality of $f(n)$. Thus we have

$$
g(f(n)) \geq n .
$$

From (9),(10), we have the desired equality.

Second, there exists a minimum solution:

$$
g(n)=n_{1}+n_{2}+\cdots+n_{t}
$$

for some $n_{1}, n_{2}, \ldots, n_{t}, t \in N$ satisfying

$$
n_{1} n_{2} \cdots n_{t} \geq n
$$

This implies $f(g(n)) \geq n$. 
(ii) Since

$$
f(3 m)=3^{m}, \quad f(3 m+1)=4 \cdot 3^{m-1}, \quad f(3 m+2)=2 \cdot 3^{m}
$$

implies

$$
g\left(3^{m}\right)=3 m, \quad g\left(4 \cdot 3^{m-1}\right)=3 m+1, \quad g\left(2 \cdot 3^{m}\right)=3 m+2
$$

we have the desired composite equalities, respectively.

Let $h: N \rightarrow N$ be a nondecreasing function with $h(1)=1$ and $h(n)$ go to $\infty$ as so does $n$. Only in this section we use two kinds of its inverse function as follows: One is the upper-semi inverse function $h^{-1}: N \rightarrow N$

$$
h^{-1}(n):=\min \{m \in N \mid h(m) \geq n\} .
$$

The other is the lower-semi inverse function $h_{-1}: N \rightarrow N$

$$
h_{-1}(n):=\operatorname{Max}\{m \in N \mid h(m) \leq n\} .
$$

We say that a value $n \in N$ is attainable if there exists some $m \in N$ satisfying $h(m)=n$. Then we have the following properties. The proof is straightforward and therefore omitted. For the same reason, some proofs are omitted in the remainder of this paper.

LEMMA 2.4.

$$
\begin{aligned}
& \text { (i) } h^{-1}, h_{-1}: N \rightarrow N \text { are nondecreasing } \\
& \text { (ii) } \quad h_{-1}(n) \geq h^{-1}(n) \quad \text { for attainable } n \in N \\
& \text { (iii) } \quad h_{-1}(n)<h^{-1}(n) \quad \text { for nonattainable } n \in N \text {. }
\end{aligned}
$$

Furthermore, for any nonattainable $n \in N$, both $h_{-1}(n)$ and $h^{-1}(n)$ take two adjacent (neighbouring) values in $N$.

LEMMA 2.5.

$$
\begin{array}{ll}
\text { (i) } & h\left(h^{-1}(n)\right) \geq n, \quad h\left(h_{-1}(n)\right) \leq n \quad n \in N \\
\text { (ii) } & h^{-1}(h(m)) \leq m, \quad h_{-1}(h(m)) \geq m \quad m \in N .
\end{array}
$$

Moreover, we have a rather strict inverse relations as follows:

Theовем 2.6. (Strong Inverse Theorem I)

(i) $\quad f^{-1}(n)=g(n), \quad g_{-1}(n)=f(n) \quad n \in N$

$$
\text { (ii) } \quad \hat{\sigma}(n) \supseteq \pi^{*}\left(f^{-1}(n)\right) \quad n \in[6,7, \ldots), \quad \pi^{*}(n)=\hat{\sigma}\left(g_{-1}(n)\right) \quad n \in[5,6, \ldots) \text {. }
$$

Proof (i) Let $n \in N$. First, from Theorem 2.3 (Weak Inverse Theorem), we have $f(g(n)) \geq n$. This implies

$$
f^{-1}(n) \leq g(n) .
$$


On the other hand, we see that $f(m) \geq n$ implies $m \geq g(n)$. Thus we get

$$
f^{-1}(n) \geq g(n) .
$$

Then we have the desired equality.

Now we show the above-mentioned implication. Let $f(m) \geq n$. Then we have a maximum solution:

$$
f(m)=n_{1} n_{2} \cdots n_{t} \geq n
$$

for some $n_{1}, n_{2}, \ldots, n_{t}, t \in N$ satisfying

$$
n_{1}+n_{2}+\cdots+n_{t}=m
$$

Thus we get $g(n) \leq m$.

Second, from $g(f(n))=n$, we have

$$
g_{-1}(n) \geq f(n)
$$

Further we see that $g(m) \leq n$ implies $m \leq f(n)$. Thus we get

$$
g_{-1}(n) \leq f(n)
$$

Then we have the desired equality.

(ii) First take $n \geq 6$. Let $m:=f^{-1}(n)$. Then we show

$$
\pi^{*}(m) \subseteq \hat{\sigma}(n) .
$$

Let $k \in \pi^{*}(m)$. Then we have

$$
f(m)=k \times f(m-k) .
$$

Letting $p:=f(m) / k$, we get

$$
p=f(m-k)=g_{-1}(m-k) .
$$

This in turn implies

$$
k+g(p) \leq m .
$$

Since $m=f^{-1}(n)=g(n)$, we have

$$
k+g(p) \leq g(n) .
$$

On the other hand, we have $k p=f(m) \geq n$. This together with the recursiveness yields

$$
k+g(p) \geq g(n)
$$

From (23),(24), we have $k \in \hat{\sigma}(n)$.

Second, take $n \geq 5$. Let $m:=g_{-1}(n)$. Then we show

$$
\hat{\sigma}(m)=\pi^{*}(n) .
$$


Let $k \in \hat{\sigma}(m)$. Then we have

$$
g(m)=k+g(l)
$$

for some $l$ with $k l=m$. Letting $q:=g(m)-k$, we get

$$
q=g(l)=f^{-1}(l) .
$$

This in turn implies

$$
k \times f(q) \geq m .
$$

Since $m=g_{-1}(n)=f(n)$, we have

$$
k \times f(q) \geq f(n)
$$

On the other hand, from $g\left(g_{-1}(n)\right)=n$, we have $k+q=g(m)=n$. This together with the recursiveness yields

$$
k \times f(q) \leq f(n), \quad k+q=n .
$$

From (25),(26), we have $k \in \pi^{*}(n)$.

Conversely, letting $k \in \pi^{*}(n)$, we have

$$
f(n)=k \times f(n-k), \quad 1<k<n .
$$

Let $p:=f(n) / k$. Then we get

$$
p=f(n-k)=g_{-1}(n-k)
$$

This implies

$$
k+g(p) \leq n
$$

Since $n=g(m)$, we have

$$
k+g(p) \leq g(m)
$$

On the other hand, we have $k p=f(n)=g_{-1}(n)=m$. This together with the recursiveness yields

$$
k+g(p) \geq g(m)
$$

From (27),(28), we have $k \in \hat{\sigma}(m)$. This completes the proof.

Henceforth we write symbolically

$$
\begin{aligned}
& f^{-1}=g, \quad g_{-1}=f \text { on } N \\
& \hat{\sigma} \supseteq \pi^{*} \circ f^{-1} \text { on }[6,7, \ldots), \quad \pi^{*}=\hat{\sigma} \circ g_{-1} \text { on }[5,6, \ldots),
\end{aligned}
$$

where $\circ$ is the composition operator between functions. 
In fact, we have the following optimal solutions:

For $n \geq 5$ the MPP has the maximum solution

$$
\begin{aligned}
& f(n)=\left\{\begin{array}{ll}
3^{m} & \text { for } n=3 m \\
4 \cdot 3^{m-1} & \text { for } n=3 m+1 \\
2 \cdot 3^{m} & \text { for } n=3 m+2
\end{array} \quad m \in N\right. \\
& \pi^{*}(n)=\left\{\begin{array}{ll}
3 & \text { for } n=3 m \\
2,3,4 & \text { for } n=3 m+1 \\
2,3 & \text { for } n=3 m+2
\end{array} \quad m \in N\right. \\
& t^{*}(n)=\left\{\begin{array}{ll}
m & \text { for } n=3 m \\
m, m+1 & \text { for } n=3 m+1 \\
m+1 & \text { for } n=3 m+2
\end{array} \quad m \in N .\right.
\end{aligned}
$$

For $n \geq 6$ the IPP has the minimum solution as follows :

$$
\begin{aligned}
& g(n)=\left\{\begin{array}{ll}
3 m & \text { for } 2 \cdot 3^{m-1}<n \leq 3^{m} \\
3 m+1 & \text { for } 3^{m}<n \leq 4 \cdot 3^{m-1} \\
3 m+2 & \text { for } 4 \cdot 3^{m-1}<n \leq 2 \cdot 3^{m}
\end{array} \quad m \in N\right. \\
& \hat{\sigma}(n)=\left\{\begin{array}{ll}
3^{\star} & \text { for } 2 \cdot 3^{m-1}<n \leq 3^{m} \\
2,3,4^{\star} & \text { for } 3^{m}<n \leq 4 \cdot 3^{m-1} \\
2,3^{\star} & \text { for } 4 \cdot 3^{m-1}<n \leq 2 \cdot 3^{m}
\end{array} \quad m \in N\right. \\
& \hat{t}(n)=\left\{\begin{array}{ll}
m^{\star} & \text { for } 2 \cdot 3^{m-1}<n \leq 3^{m} \\
m, m+1^{\star} & \text { for } 3^{m}<n \leq 4 \cdot 3^{m-1} \\
m+1^{\star} & \text { for } 4 \cdot 3^{m-1}<n \leq 2 \cdot 3^{m}
\end{array} \quad m \in N,\right.
\end{aligned}
$$

where, means that the specification is a minimal expression (subset) of $\hat{\sigma}(n)$ and a possibility of $\hat{t}(n)$. However, for the cases $n=3^{m}, 4 \cdot 3^{m-1}, 2 \cdot 3^{m}$, it specifies the complete expression. The full description of $\hat{\sigma}(n)$ is given in Figures $2.1,2.2, \ldots, 2.6$.

We are interested in the ultimate distribution of average numbers of optimal (maximum and minimum) partitions

$$
\frac{n}{t^{*}(n)} \quad \text { and } \quad n^{1 / \hat{t}(n)}
$$

for the limitting case $n \rightarrow \infty$, respectively. Both will converge to a common Euler number $e$.

\subsection{Fixed Partition Problems}

In this subsection we consider, for any $k \in N$, the main partition problem:

$$
\begin{array}{lll}
\text { Maximize } & n_{1} n_{2} \cdots n_{k} \\
\operatorname{MPP}(n: k) & \text { subject to } & \text { (i) } n_{1}+n_{2}+\cdots+n_{k}=n \\
& \text { (ii) } n_{1}, n_{2}, \ldots, n_{k} \in N
\end{array}
$$


where $k \leq n \in N$ and, its inverse partition problem:

$$
\begin{array}{lll}
\operatorname{minimize} & n_{1}+n_{2}+\cdots+n_{k} \\
\operatorname{IPP}(n ; k) & \text { subject to } & \left(\text { i) }{ }^{\prime} \quad n_{1} n_{2} \cdots n_{k} \geq n\right. \\
& \text { (ii) } n_{1}, n_{2}, \ldots, n_{k} \in N
\end{array}
$$

where $n \in N$.

Let $f_{k}(n), g_{k}(n)$ be the maximum value and the minimum value, respectively. Then

LEMma 2.7. The maximum value functions $f_{k}:[k, k+1, \ldots) \rightarrow N$ are strictly increasing but $g_{k}: N \rightarrow N$ are nondecreasing. They go to $\infty$ as so does $n$.

THEOREM 2.8 .

$$
\begin{aligned}
& f_{k}(n)=\operatorname{Max}_{1 \leq m<n}\left[m f_{k-1}(n-m)\right] \quad k \geq 2 \\
& f_{1}(n)=n \quad n \in N \\
& g_{k}(n)=\min _{\substack{1<m l<n \\
m l \geq n}}\left[m+g_{k-1}(l)\right] \quad k \geq 2 \\
& g_{1}(n)=n \quad n \in N .
\end{aligned}
$$

The minimization is restricted to the set of all pairs $(m, l)$ satisfying the condition.

Let $\pi_{k}^{*}(n)$ be the minimizer in $(40)$ and $\hat{\sigma}_{k}(n)$ the value of $m$ for the maximizer in (42), respectively.

We have an envelopping relation between the regular problem and the fixed problems:

Theorem 2.9. (Envelopping Theorem)

$$
\begin{aligned}
& f(n)=\operatorname{Max}_{k \geq 1} f_{k}(n) \quad n \in N \\
& g(n)=\min _{k \geq 1} g_{k}(n) \quad n \in N .
\end{aligned}
$$

Furthermore, we have the inverse relationship between main and inverse fixed partition problems:

Theorem 2.10. (Inverse Theorem)

(i) $g_{k}=f_{k}^{-1}$ on $N, f_{k}=\left(g_{k}\right)_{-1}$ on $[k, k+1, \ldots), k \geq 1$

(ii) $\hat{\sigma}_{k} \supseteq \pi_{k}^{*} \circ f_{k}^{-1}$ on $N, \quad \pi_{k}^{*}=\hat{\sigma}_{k} \circ\left(g_{k}\right)_{-1} \quad$ on $[k, k+1, \ldots), k \geq 2 .(47)$

\section{Finitely Many Partition of Continuum}

In this section we are concerned with partition of any given nonnegative real into finitely many ones. 


\subsection{Regular Partition Problems}

We consider the main partition problem:

$$
\begin{array}{lll}
\text { Maximize } & x_{1} x_{2} \cdots x_{t} \\
\operatorname{MPP}(c) & \text { (i) } x_{1}+x_{2}+\cdots+x_{t}=c \\
& \text { (ii) } x_{m} \geq 0 \quad 1 \leq m \leq t \\
& \text { (iii) } t \geq 1
\end{array}
$$

and the inverse partition problem:

$$
\begin{array}{lll}
\text { minimize } & x_{1}+x_{2}+\cdots+x_{t} \\
\operatorname{IPP}(c) \quad & \text { (i) } x_{1} x_{2} \cdots x_{t}=c \\
& \text { (ii) } x_{m} \geq 0 \quad 1 \leq m \leq t \\
& \text { (iii) } t \geq 1
\end{array}
$$

where $c \geq 0$. The three constraints mean to partition a given nonnegative real $c$ into $t$ nonnegative ones, which is also a control variable.

Let $f(c), g(c)$ be the maximum value and the minimu value, respectively. Then we have

Lemma 3.1. Both optimum value functions $f, g:[0, \infty) \rightarrow[0, \infty)$ are continuous and strictly increasing. Both go to $\infty$ as so does $c$.

THEOREM 3.2 .

$$
\begin{aligned}
f(c) & =\operatorname{Max}_{0 \leq x \leq c}[x f(c-x)] \quad c \geq 4 \\
f(c) & =c \quad 0 \leq c \leq 4 \\
g(c) & =\min _{0<x<\infty}\left[x+g\left(\frac{c}{x}\right)\right] \quad c \geq 4 \\
g(c) & =c \quad 0 \leq c \leq 4 .
\end{aligned}
$$

Let us define $\pi^{*}(c), \hat{\sigma}(c)$ be the maximizer in (50) and the minimizer in (52), respectively.

Let $h^{-1}$ be the inverse function of a continuous strictly increasing function $h$.

Then we have the inverse relationship between Main and Inverse Divisin Problems:

Theorem 3.3. (Inverse Theorem)

$$
\begin{aligned}
& \text { (i) } g=f^{-1}, f=g^{-1} \text { on }[0, \infty) \\
& \text { (ii) } \hat{\sigma}=\pi^{*} \circ f^{-1}, \quad \pi^{*}=\hat{\sigma} \circ g^{-1} \text { on }[4, \infty) \text {. }
\end{aligned}
$$

In fact, we see that both problems have the optimal solutions as follows:

$$
\begin{gathered}
f(c)=\left(\frac{c}{n}\right)^{n} \quad c \in I_{n}, \quad n \geq 1 \\
\pi^{*}(c)=\frac{c}{n}, t^{*}(c)=n \quad c \in I_{n}, \quad n \geq 2
\end{gathered}
$$


where $I_{n}$ is the interval

$$
\begin{gathered}
I_{1}=[0,4], \quad I_{n}=\left[\frac{n^{n}}{(n-1)^{n-1}}, \frac{(n+1)^{n+1}}{n^{n}}\right] \quad n \geq 2 . \\
g(c)=n c^{1 / n} \quad c \in J_{n}, \quad n \geq 1 \\
\hat{\sigma}(c)=c^{1 / n}, \hat{t}(c)=n \quad c \in J_{n}, \quad n \geq 2
\end{gathered}
$$

where $J_{n}$ is the interval

$$
J_{1}=[0,4], \quad J_{n}=\left[\left(\frac{n}{n-1}\right)^{(n-1) n},\left(\frac{n+1}{n}\right)^{n(n+1)}\right] n \geq 2 .
$$

We note that

$$
\lim _{c \rightarrow \infty} \frac{c}{t^{*}(c)}=e, \quad \lim _{c \rightarrow \infty} c^{1 / \hat{t}(c)}=e
$$

\subsection{Fixed Partition Problems}

In this subsection we are concerned with partition into any given total number $n$. For any $n \in N$, we consider the main partition problem and the inverse partition problem:

$$
\begin{array}{cll} 
& \text { Maximize } & x_{1} x_{2} \cdots x_{n} \\
\operatorname{MPP}(c ; n) & \text { (i) } x_{1}+x_{2}+\cdots+x_{n}=c \\
& \text { (ii) } x_{i} \geq 0 \quad 1 \leq i \leq n \\
& \\
\operatorname{IPP}(c ; n) & \text { minimizect to } & x_{1}+x_{2}+\cdots+x_{n} \\
& \text { subject to } & \text { (i) } x_{1} x_{2} \cdots x_{n} \geq c \\
& & \text { (ii) } x_{i} \geq 0 \quad 1 \leq i \leq n
\end{array}
$$

where $c \in[0, \infty), n \in N$. Let $f_{n}(c), g_{n}(c)$ be the maximum value and minimum value, respectively. Then

LEMMA 3.4. Both optimum value functions $f_{n}, g_{n}:[0, \infty) \rightarrow[0, \infty)$ are continuous and strictly increasing. They go to $\infty$ as so does $c$.

THEOREM 3.5 .

$$
\begin{aligned}
f_{n}(c) & =\operatorname{Max}_{0 \leq x \leq c}\left[x f_{n-1}(c-x)\right] \quad c \in[0, \infty), \quad n \geq 2 \\
f_{1}(c) & =c \quad c \in[0, \infty) \\
g_{n}(c) & =\min _{0<x<\infty}\left[x+g_{n-1}\left(\frac{c}{x}\right)\right] \quad c \in[0, \infty), \quad n \geq 2 \\
g_{1}(c) & =c \quad c \in[0, \infty) .
\end{aligned}
$$


We denote the maximizer in $(65)$ by $\pi_{n}^{*}(c)$ and the minimizer in $(67)$ by $\hat{\sigma}_{n}(c)$, respectively.

We have also the envelopping relation and the inverse relations as follows:

THEOREM 3.6.

$$
\begin{aligned}
& f(c)=\operatorname{Max}_{n \geq 1} f_{n}(c) \quad c \in[0, \infty) \\
& g(c)=\min _{n \geq 1} g_{n}(c) \quad c \in[0, \infty) .
\end{aligned}
$$

TheOREM 3.7. (Inverse Theorem (Iwamoto $(1977 a, 1977 b, 1977 c)$ )

(i) $g_{n}=f_{n}^{-1}, f_{n}=g_{n}^{-1}$ on $[0, \infty), n \geq 1$

(ii) $\hat{\sigma}_{n}=\pi_{n}^{*} \circ f_{n}^{-1}, \pi_{n}^{*}=\hat{\sigma}_{n} \circ g_{n}^{-1}$ on $[0, \infty), n \geq 2$.

In fact, the $\operatorname{MPP}(c ; n)$ has the following optimal solutions:

$$
\begin{aligned}
& f_{n}(c)=\left(\frac{c}{n}\right)^{n} \quad c \in[0, \infty), \quad n \geq 1 \\
& \pi_{n}^{*}(c)=\frac{c}{n} \quad c \in[0, \infty), \quad n \geq 2 .
\end{aligned}
$$

The $\operatorname{IPP}(c ; n)$ has the optimal solutions:

$$
\begin{array}{ll}
g_{n}(c)=n c^{1 / n} & c \in[0, \infty), \quad n \geq 1 \\
\hat{\sigma}_{n}(c)=c^{1 / n} & c \in[0, \infty), \quad n \geq 2 .
\end{array}
$$

\section{Continuously Many Partition of Continuum}

In this section we are concened with a continuous partition of any nonnegative real into any continuously many ones (see Iwamoto(1983), Iwamoto and Wang(1983,1986)).

\subsection{Regular Partition Problems}

As regular continuous partition, we consider the main partition problem:

$$
\begin{aligned}
& \text { Maximize } \quad \int_{0}^{t} \log x(s) d s \\
& \operatorname{MCPP}(c) \quad \text { subject to (i) } \int_{0}^{t} x(s) d s=c \\
& \text { (ii) } x(s) \geq 0 \quad 0 \leq s \leq t \\
& \text { (iii) } t \geq 0
\end{aligned}
$$

and the inverse partition problem

$$
\begin{aligned}
& \operatorname{minimize} \int_{0}^{t} x(s) d s \\
& \operatorname{ICPP}(c) \\
& \text { subject to } \\
& \text { (i) } \int_{0}^{t} \log x(s) d s=c \\
& \text { (ii) } x(s) \geq 0 \quad 0 \leq s \leq t \\
& \text { (iii) } t \geq 0
\end{aligned}
$$


where $c \geq 0$.

Let $f(c), g(c)$ be the maximum value and the minimum value, respectively. First we have the strict monotonicity of optimum value functions $f(\cdot), g(\cdot)$ as follows:

Lemma 4.1. The optimum value functions $f, g:[0, \infty) \rightarrow[0, \infty)$ are differentiable and strictly increasing. Both go to $\infty$ as so does $c$.

THEOREM 4.2. Both the optimum value functions $f=f(c), g=g(c)$ satisfy the Bellman equations:

$$
\begin{aligned}
& 0=\operatorname{Max}_{x \geq 0}\left[\log x-f^{\prime} x\right], \quad f(0)=0 \\
& 0=\min _{x \geq 0}\left[x-g^{\prime} \log x\right], \quad g(0)=0,
\end{aligned}
$$

respectively.

Letting $\pi^{*}(c)$ be the maximizer of $(79)$ and $\hat{\sigma}(c)$ be the minimizer of $(80)$, we have

$$
\begin{array}{ll}
\log f^{\prime}+1=0, & \text { for } x^{*}=\pi^{*}(c)=1 / f^{\prime}(c) . \\
\log g^{\prime}-1=0, & \text { for } \hat{x}=\hat{\sigma}(c)=g^{\prime}(c),
\end{array}
$$

respectively. Thus we have optimum value functions, optimizer and optimum continuous partition with optimum length as follows:

$$
\begin{aligned}
& f(c)=\frac{c}{e}, \quad \pi^{*}(c)=e ; \quad x^{*}(s)=e, \quad t^{*}(c)=\frac{c}{e} \\
& g(c)=e c, \quad \hat{\sigma}(c)=e ; \quad \hat{x}(s)=e, \quad \hat{t}(c)=c .
\end{aligned}
$$

Furthermore, we have the inverse relationship between Main and Inverse Partition Problems:

THEOREM 4.3. (Inverse Theorem)

$$
\begin{array}{ll}
\text { (i) } & g=f^{-1}, f=g^{-1} \\
\text { (ii) } & \hat{\sigma}=\pi^{*} \circ f^{-1}, \quad \pi^{*}=\hat{\sigma} \circ g^{-1} \\
\text { (iii) } & \hat{t}=t^{*} \circ f^{-1}, \quad t^{*}=\hat{t} \circ g^{-1} .
\end{array}
$$

We remark that

$$
\frac{c}{t^{*}(c)}=e, \quad c^{1 / \hat{t}(\log c)}=e
$$

\subsection{Fixed Partition Problems}

For any given real number $t \geq 0$, we consider the pair of maximization problem and minimization problem (Iwamoto and Wang $(1983,1986)$ ):

$$
\begin{array}{lll} 
& \text { Maximize } & \int_{0}^{t} \log x(s) d s \\
\operatorname{MCPP}(c ; t) \quad \text { subject to } & \text { (i) } \int_{0}^{t} x(s) d s=c \\
& \text { (ii) } x(s) \geq 0 \quad 0 \leq s \leq t
\end{array}
$$


and

$$
\begin{array}{lll} 
& \text { minimize } & \int_{0}^{t} x(s) d s \\
\operatorname{ICPP}(c ; t) \quad \text { subject to } & (\mathrm{i})^{\prime} \int_{0}^{t} \log x(s) d s=c \\
& \text { (ii) } x(s) \geq 0 \quad 0 \leq s \leq t
\end{array}
$$

where $c \geq 0$.

Let $f(t, c), g(t, c)$ be the maximum value and the minimum value, respectively. First we have the strict monotonicity of optimum value functions $f(t, \cdot), g(t, \cdot)$ as follows:

LEмma 4.4. The optimum value functions $f=f(t, c), g=g(t, c)$ are continuously differentiable. For each $t \geq 0$, both functions $f(t, \cdot), g(t, \cdot):[0, \infty) \rightarrow[0, \infty)$ are strictly increasing. Both go to $\infty$ as so does $c$.

TheOREM 4.5. Both the optimum value functions $f=f(t, c), g=g(t, c)$ satisfy the Bellman equations:

$$
\begin{aligned}
& f_{t}=\operatorname{Max}_{x \geq 0}\left[\log x-f_{c} x\right], \quad f(0, c)=0 \\
& g_{t}=\min _{x \geq 0}\left[x-g_{c} \log x\right], \quad g(0, c)=0,
\end{aligned}
$$

respectively.

Letting $\pi^{*}(t, c)$ be the maximizer of $(91)$ and $\hat{\sigma}(t, c)$ be the minimizer of $(92)$, we have

$$
\begin{aligned}
& f_{t}+\log f_{c}+1=0, \quad \text { for } x^{*}=\pi^{*}(t, c)=1 / f_{c}(t, c) \\
& g_{t}=g_{c}\left(1-\log g_{c}\right), \quad \text { for } \hat{x}=\hat{\sigma}(t, c)=g_{c}(t, c),
\end{aligned}
$$

respectively. Thus we have optimum value function, optimizer and optimum partition number as follows:

$$
\begin{aligned}
& f(t, c)=t \log \frac{c}{t}, \quad \pi^{*}(t, c)=\frac{c}{t} ; \quad x^{*}(s)=\frac{c}{t} \\
& g(t, c)=t e^{c / t}, \quad \hat{\sigma}(t, c)=e^{c / t} ; \quad \hat{x}(s)=e^{c / t} .
\end{aligned}
$$

Furthermore, we have an envelopping relation between the regular problem and the fixed problems:

TheOREM 4.6. (Envelopping Theorem)

$$
\begin{array}{ll}
f(c)=\operatorname{Max}_{t \geq 0} f(c, t) & c \in[0, \infty) \\
g(c)=\min _{t \geq 0} g(c, t) & c \in[0, \infty) .
\end{array}
$$


By letting $h_{t}(c):=h(t, c)$, we have the inverse relationship between Main and Inverse Partition Problems:

THEOREM 4.7. (Inverse Theorem)

$$
\begin{array}{ll}
\text { (i) } g_{t} & =f_{t}^{-1}, f_{t}=g_{t}^{-1} \\
\text { (ii) } \hat{\sigma}_{t}=\pi_{t}^{*} \circ f_{t}^{-1}, \quad \pi_{t}^{*}=\hat{\sigma}_{t} \circ g_{t}^{-1} .
\end{array}
$$

\section{Optimal Solution Tables and Figures}

In this section we specify optimal solutions in tables. Optimal value function, optimal policy and optimal partition are illustrated. Further the forementioned inverse relation are also shown in tables. The specification verifies that all the results in Inverese Theorems are valid.

Table 1. Optimal Solution for MPP

\begin{tabular}{|c|cll|}
\hline given & maximum & optimal & optimal \\
quantity & value & policy & partition \\
$n$ & $f(n)$ & $\pi^{*}(n)$ & $n=$ \\
\hline \hline 1 & 1 & - & $1=1$ \\
2 & 2 & - & $2=2$ \\
3 & 3 & - & $3=3$ \\
4 & 4 & - & $4=4=2+2$ \\
5 & 6 & 2,3 & $5=3+2$ \\
\hline 6 & 9 & 3 & $6=3+3$ \\
7 & 12 & $2,3,4$ & $7=3+2+2=3+4$ \\
8 & 18 & 2,3 & $8=3+3+2$ \\
\hline 9 & 27 & 3 & $9=3+3+3$ \\
10 & 36 & $2,3,4$ & $10=3+3+2+2=3+3+4$ \\
11 & 54 & 2,3 & $11=3+3+3+2$ \\
\hline 12 & 81 & 3 & $12=3+3+3+3$ \\
13 & 108 & $2,3,4$ & $13=3+3+3+2+2=3+3+3+4$ \\
14 & 162 & 2,3 & $14=3+3+3+3+2$ \\
\hline 15 & 243 & 3 & $15=3+3+3+3+3$ \\
16 & 324 & $2,3,4$ & $16=3+3+3+3+2+2=3+3+3+3+4$ \\
17 & 486 & 2,3 & $17=3+3+3+3+3+2$ \\
\hline$\vdots$ & $\vdots$ & $\vdots$ & $\vdots$ \\
\hline $3 m$ & $3^{m}$ & 3 & $3 m=3+\cdots+3$ \\
$3 m+1$ & $4 \cdot 3^{m-1}$ & $2,3,4$ & $3 m+1=3+\cdots+3+2+2=3+\cdots+3+4$ \\
$3 m+2$ & $2 \cdot 3^{m}$ & 2,3 & $3 m+2=3+\cdots+3+2$ \\
\hline$\vdots$ & $\vdots$ & $\vdots$ & $\vdots$ \\
\hline
\end{tabular}


Table 2.1 Optimal Solution for IPP

\begin{tabular}{|c|c|c|c|}
\hline $\begin{array}{c}\text { given } \\
\text { quantity } \\
n\end{array}$ & $\begin{array}{l}\text { minimum } \\
\text { value } \\
g(n) \\
\end{array}$ & $\begin{array}{l}\text { optimal } \\
\text { policy } \\
\hat{\sigma}(n)\end{array}$ & $\begin{array}{l}\text { optimal } \\
\text { partition } \\
\qquad \geq n(=n \text { or }>n)\end{array}$ \\
\hline 1 & 1 & - & $1=1$ \\
\hline 2 & 2 & - & $2=2$ \\
\hline 3 & 3 & - & $3=3$ \\
\hline 4 & 4 & - & $2^{2}=4=4$ \\
\hline 5 & 5 & - & $3 \cdot 2>5=5$ \\
\hline 6 & 5 & 2,3 & $3 \cdot 2=6$ \\
\hline 7 & 6 & $2,3,4$ & $3^{2}>2^{3}>7$ \\
\hline 8 & 6 & $2,3,4$ & $3^{2}>2^{3}=8$ \\
\hline 9 & 6 & 3 & $3^{2}=9$ \\
\hline 10 & 7 & $2,3,4,5$ & $3 \cdot 2^{2}>5 \cdot 2=10$ \\
\hline 11 & 7 & $2,3,4$ & $3 \cdot 2^{2}>11$ \\
\hline 12 & 7 & $2,3,4$ & $3 \cdot 2^{2}=12$ \\
\hline 13 & 8 & $2,3,4,5$ & $3^{2} \cdot 2>2^{4}>5 \cdot 3>13$ \\
\hline 14 & 8 & $2,3,4,5$ & $3^{2} \cdot 2>2^{4}>5 \cdot 3>14$ \\
\hline 15 & 8 & $2,3,4,5$ & $3^{2} \cdot 2>2^{4}>5 \cdot 3=15$ \\
\hline 16 & 8 & $2,3,4$ & $3^{2} \cdot 2>2^{4}=16$ \\
\hline 17 & 8 & 2,3 & $3^{2} \cdot 2>17$ \\
\hline 18 & 8 & 2,3 & $3^{2} \cdot 2=18$ \\
\hline 19 & 9 & $2,3,4,5$ & $3^{3}>3 \cdot 2^{3}>5 \cdot 2^{2}>19$ \\
\hline 20 & 9 & $2,3,4,5$ & $3^{3}>3 \cdot 2^{3}>5 \cdot 2^{2}=20$ \\
\hline 21 & 9 & $2,3,4$ & $3^{3}>3 \cdot 2^{3}>21$ \\
\hline 22 & 9 & $2,3,4$ & $3^{3}>3 \cdot 2^{3}>22$ \\
\hline 23 & 9 & $2,3,4$ & $3^{3}>3 \cdot 2^{3}>23$ \\
\hline 24 & 9 & $2,3,4$ & $3^{3}>3 \cdot 2^{3}=24$ \\
\hline 25 & 9 & 3 & $3^{3}>25$ \\
\hline 26 & 9 & 3 & $3^{3}>26$ \\
\hline 27 & 9 & 3 & $3^{3}=27$ \\
\hline 28 & 10 & $2,3,4,5$ & $3^{2} \cdot 2^{2}>2^{5}>5 \cdot 3 \cdot 2>28$ \\
\hline 29 & 10 & $2,3,4,5$ & $3^{2} \cdot 2^{2}>2^{5}>5 \cdot 3 \cdot 2>29$ \\
\hline 30 & 10 & $2,3,4,5$ & $3^{2} \cdot 2^{2}>2^{5}>5 \cdot 3 \cdot 2=30$ \\
\hline 31 & 10 & $2,3,4$ & $3^{2} \cdot 2^{2}>2^{5}>31$ \\
\hline 32 & 10 & $2,3,4$ & $3^{2} \cdot 2^{2}>2^{5}=32$ \\
\hline 33 & 10 & $2,3,4$ & $3^{2} \cdot 2^{2}>33$ \\
\hline 34 & 10 & $2,3,4$ & $3^{2} \cdot 2^{2}>34$ \\
\hline 35 & 10 & $2,3,4$ & $3^{2} \cdot 2^{2}>35$ \\
\hline 36 & 10 & $2,3,4$ & $3^{2} \cdot 2^{2}=36$ \\
\hline
\end{tabular}


Table 2.2 Optimal Solution for IPP

\begin{tabular}{|c|c|c|c|}
\hline $\begin{array}{c}\text { given } \\
\text { quantity } \\
n\end{array}$ & $\begin{array}{l}\text { minimum } \\
\text { value } \\
g(n)\end{array}$ & $\begin{array}{l}\text { optimal } \\
\text { policy } \\
\hat{\sigma}(n)\end{array}$ & $\begin{array}{l}\text { optimal } \\
\text { partition } \\
\qquad \geq n(=n \text { or }>n)\end{array}$ \\
\hline 36 & 10 & $2,2,3,4$ & $3^{2} \cdot 2^{2}=36$ \\
\hline 37 & 11 & $2,3,4,5$ & $3^{3} \cdot 2>3 \cdot 2^{4}>5 \cdot 3^{2}>5 \cdot 2^{3}>37$ \\
\hline 38 & 11 & $2,3,4,5$ & $3^{3} \cdot 2>3 \cdot 2^{4}>5 \cdot 3^{2}>5 \cdot 2^{3}>38$ \\
\hline 39 & 11 & $2,3,4,5$ & $3^{3} \cdot 2>3 \cdot 2^{4}>5 \cdot 3^{2}>5 \cdot 2^{3}>39$ \\
\hline 40 & 11 & $2,3,4,5$ & $3^{3} \cdot 2>3 \cdot 2^{4}>5 \cdot 3^{2}>5 \cdot 2^{3}=40$ \\
\hline 41 & 11 & $2,3,4,5$ & $3^{3} \cdot 2>3 \cdot 2^{4}>5 \cdot 3^{2}>41$ \\
\hline 42 & 11 & $2,3,4,5$ & $3^{3} \cdot 2>3 \cdot 2^{4}>5 \cdot 3^{2}>42$ \\
\hline 43 & 11 & $2,3,4,5$ & $3^{3} \cdot 2>3 \cdot 2^{4}>5 \cdot 3^{2}>43$ \\
\hline 44 & 11 & $2,3,4,5$ & $3^{3} \cdot 2>3 \cdot 2^{4}>5 \cdot 3^{2}>44$ \\
\hline 45 & 11 & $2,3,4,5$ & $3^{3} \cdot 2>3 \cdot 2^{4}>5 \cdot 3^{2}=45$ \\
\hline 46 & 11 & $2,3,4$ & $3^{3} \cdot 2>3 \cdot 2^{4}>46$ \\
\hline 47 & 11 & $2,3,4$ & $3^{3} \cdot 2>3 \cdot 2^{4}>47$ \\
\hline 48 & 11 & $2,3,4$ & $3^{3} \cdot 2>3 \cdot 2^{4}=48$ \\
\hline 49 & 11 & 2,3 & $3^{3} \cdot 2>49$ \\
\hline 50 & 11 & 2,3 & $3^{3} \cdot 2>50$ \\
\hline$\vdots$ & $\vdots$ & $\vdots$ & $\vdots$ \\
\hline 53 & 11 & 2,3 & $3^{3} \cdot 2>53$ \\
\hline 54 & 11 & 2,3 & $3^{3} \cdot 2=54$ \\
\hline 55 & 12 & $2,3,4,5$ & $3^{4}>3^{2} \cdot 2^{3}>2^{6}>5 \cdot 3 \cdot 2^{2}>55$ \\
\hline 56 & 12 & $2,3,4,5$ & $3^{4}>3^{2} \cdot 2^{3}>2^{6}>5 \cdot 3 \cdot 2^{2}>56$ \\
\hline$\vdots$ & $\vdots$ & $\vdots$ & $\vdots$ \\
\hline 59 & 12 & $2,3,4,5$ & $3^{4}>3^{2} \cdot 2^{3}>2^{6}>5 \cdot 3 \cdot 2^{2}>59$ \\
\hline 60 & 12 & $2,3,4,5$ & $3^{4}>3^{2} \cdot 2^{3}>2^{6}>5 \cdot 3 \cdot 2^{2}=60$ \\
\hline 61 & 12 & $2,3,4$ & $3^{4}>3^{2} \cdot 2^{3}>2^{6}>61$ \\
\hline : & $\vdots$ & $\vdots$ & $\vdots \quad($ continued $)$ \\
\hline
\end{tabular}


Table 2.3 Optimal Solution for IPP

\begin{tabular}{|c|c|c|c|}
\hline $\begin{array}{c}\text { given } \\
\text { quantity } \\
n \\
\end{array}$ & $\begin{array}{c}\text { minimum } \\
\text { value } \\
g(n) \\
\end{array}$ & $\begin{array}{l}\text { optimal } \\
\text { policy } \\
\hat{\sigma}(n) \\
\end{array}$ & $\begin{array}{l}\text { optimal } \\
\text { partition } \\
\geq \geq n(=n \text { or }>n)\end{array}$ \\
\hline$\vdots$ & $\vdots$ & $\vdots$ & $\vdots$ \\
\hline 60 & 12 & $2,3,4,5$ & $3^{4}>3^{2} \cdot 2^{3}>2^{6}>5 \cdot 3 \cdot 2^{2}=60$ \\
\hline 61 & 12 & $2,3,4$ & $3^{4}>3^{2} \cdot 2^{3}>2^{6}>61$ \\
\hline 62 & 12 & $2,3,4$ & $3^{4}>3^{2} \cdot 2^{3}>2^{6}>62$ \\
\hline 63 & 12 & $2,3,4$ & $3^{4}>3^{2} \cdot 2^{3}>2^{6}>63$ \\
\hline 64 & 12 & $2,3,4$ & $3^{4}>3^{2} \cdot 2^{3}>2^{6}=64$ \\
\hline 65 & 12 & $2,3,4$ & $3^{4}>3^{2} \cdot 2^{3}>65$ \\
\hline 66 & 12 & $2,3,4$ & $3^{4}>3^{2} \cdot 2^{3}>66$ \\
\hline$\vdots$ & $\vdots$ & $\vdots$ & $\vdots$ \\
\hline 71 & 12 & $2,3,4$ & $3^{4}>3^{2} \cdot 2^{3}>71$ \\
\hline 72 & 12 & $2,3,4$ & $3^{4}>3^{2} \cdot 2^{3}=72$ \\
\hline 73 & 12 & 3 & $3^{4}>73$ \\
\hline 74 & 12 & 3 & $3^{4}>74$ \\
\hline$\vdots$ & $\vdots$ & $\vdots$ & $\vdots$ \\
\hline 80 & 12 & 3 & $3^{4}>80$ \\
\hline 81 & 12 & 3 & $3^{4}=81$ \\
\hline 82 & 13 & $2,3,4,5$ & $3^{3} \cdot 2^{2}>3 \cdot 2^{5}>5 \cdot 3^{2} \cdot 2>82$ \\
\hline 83 & 13 & $2,3,4,5$ & $3^{3} \cdot 2^{2}>3 \cdot 2^{5}>5 \cdot 3^{2} \cdot 2>83$ \\
\hline$\vdots$ & $\vdots$ & $\vdots$ & $\vdots$ \\
\hline 89 & 13 & $2,3,4,5$ & $3^{3} \cdot 2^{2}>3 \cdot 2^{5}>5 \cdot 3^{2} \cdot 2>89$ \\
\hline 90 & 13 & $2,3,4,5$ & $3^{3} \cdot 2^{2}>3 \cdot 2^{5}>5 \cdot 3^{2} \cdot 2=90$ \\
\hline 91 & 13 & $2,3,4$ & $3^{3} \cdot 2^{2}>3 \cdot 2^{5}>91$ \\
\hline 92 & 13 & $2,3,4$ & $3^{3} \cdot 2^{2}>3 \cdot 2^{5}>92$ \\
\hline$\vdots$ & $\vdots$ & $\vdots$ & $\vdots$ \\
\hline 95 & 13 & $2,3,4$ & $3^{3} \cdot 2^{2}>3 \cdot 2^{5}>95$ \\
\hline 96 & 13 & $2,3,4$ & $3^{3} \cdot 2^{2}>3 \cdot 2^{5}=96$ \\
\hline 97 & 13 & $2,3,4$ & $3^{3} \cdot 2^{2}>97$ \\
\hline 98 & 13 & $2,3,4$ & $3^{3} \cdot 2^{2}>98$ \\
\hline$\vdots$ & $\vdots$ & $\vdots$ & $\vdots$ \\
\hline 107 & 13 & $2,3,4$ & $3^{3} \cdot 2^{2}>107$ \\
\hline 108 & 13 & $2,3,4$ & $3^{3} \cdot 2^{2}=108$ \\
\hline$\vdots$ & $\vdots$ & $\vdots$ & (continued) \\
\hline
\end{tabular}


Table 2.4 Optimal Solution for IPP

\begin{tabular}{|c|c|c|c|}
\hline $\begin{array}{c}\text { given } \\
\text { quantity } \\
n\end{array}$ & $\begin{array}{c}\text { minimum } \\
\text { value } \\
g(n) \\
\end{array}$ & $\begin{array}{l}\text { optimal } \\
\text { policy } \\
\hat{\sigma}(n) \\
\end{array}$ & $\begin{array}{l}\text { optimal } \\
\text { partition } \\
\qquad \geq n(=n \text { or }>n)\end{array}$ \\
\hline 108 & 13 & $2,3,4$ & $3^{3} \cdot 2^{2}=108$ \\
\hline$\vdots$ & : & $\vdots$ & $\vdots$ \\
\hline $3^{m}+1$ & $3 m+1$ & $2,3,4,5$ & $\begin{array}{l}3^{m-1} \cdot 2^{2}>3^{m-3} \cdot 2^{5}>5 \cdot 3^{m-2} \cdot 2 \\
>3^{m-5} \cdot 2^{8}>3^{m}+1\end{array}$ \\
\hline $3^{m}+2$ & $3 m+1$ & $2,3,4,5$ & $\begin{array}{l}3^{m-1} \cdot 2^{2}>3^{m-3} \cdot 2^{5}>5 \cdot 3^{m-2} \cdot 2 \\
>3^{m-5} \cdot 2^{8}>3^{m}+2\end{array}$ \\
\hline$\vdots$ & $\vdots$ & $\vdots$ & $\vdots$ \\
\hline $3^{m-5} \cdot 2^{8}$ & $3 m+1$ & $2,3,4,5$ & $\begin{array}{l}3^{m-1} \cdot 2^{2}>3^{m-3} \cdot 2^{5}>5 \cdot 3^{m-2} \cdot 2 \\
>3^{m-5} \cdot 2^{8}=3^{m-5} \cdot 2^{8}\end{array}$ \\
\hline $3^{m-5} \cdot 2^{8}+1$ & $3 m+1$ & $2,3,4,5$ & $\begin{array}{l}3^{m-1} \cdot 2^{2}>3^{m-3} \cdot 2^{5}>5 \cdot 3^{m-2} \cdot 2 \\
>3^{m-5} \cdot 2^{8}+1\end{array}$ \\
\hline $3^{m-5} \cdot 2^{8}+2$ & $3 m+1$ & $2,3,4,5$ & $\begin{array}{l}3^{m-1} \cdot 2^{2}>3^{m-3} \cdot 2^{5}>5 \cdot 3^{m-2} \cdot 2 \\
>3^{m-5} \cdot 2^{8}+2\end{array}$ \\
\hline$\vdots$ & $\vdots$ & $\vdots$ & $\vdots$ \\
\hline $5 \cdot 3^{m-2} \cdot 2$ & $3 m+1$ & $2,3,4,5$ & $\begin{array}{l}3^{m-1} \cdot 2^{2}>3^{m-3} \cdot 2^{5}>5 \cdot 3^{m-2} \cdot 2 \\
=5 \cdot 3^{m-2} \cdot 2\end{array}$ \\
\hline $5 \cdot 3^{m-2} \cdot 2+1$ & $3 m+1$ & $2,3,4$ & $3^{m-1} \cdot 2^{2}>3^{m-3} \cdot 2^{5}+1$ \\
\hline $5 \cdot 3^{m-2} \cdot 2+2$ & $3 m+1$ & $2,3,4$ & $3^{m-1} \cdot 2^{2}>3^{m-3} \cdot 2^{5}>5 \cdot 3^{m-2} \cdot 2+2$ \\
\hline$\vdots$ & $\vdots$ & $\vdots$ & $\vdots$ \\
\hline $3^{m-3} \cdot 2^{5}$ & $3 m+1$ & $2,3,4$ & $3^{m-1} \cdot 2^{2}>3^{m-3} \cdot 2^{5}=3^{m-3} \cdot 2^{5}$ \\
\hline $3^{m-3} \cdot 2^{5}+1$ & $3 m+1$ & $2,3,4$ & $3^{m-1} \cdot 2^{2}>3^{m-3} \cdot 2^{5}+1$ \\
\hline $3^{m-3} \cdot 2^{5}+2$ & $3 m+1$ & $2,3,4$ & $3^{m-1} \cdot 2^{2}>3^{m-3} \cdot 2^{5}+2$ \\
\hline$\vdots$ & $\vdots$ & $\vdots$ & $\vdots$ \\
\hline $3^{m-1} \cdot 2^{2}$ & $3 m+1$ & $2,3,4$ & $3^{m-1} \cdot 2^{2}=3^{m-1} \cdot 2^{2}$ \\
\hline $3^{m-1} \cdot 2^{2}+1$ & $3 m+2$ & $2,3,4,5$ & $\begin{array}{l}3^{m} \cdot 2>3^{m-2} \cdot 2^{4}>5 \cdot 3^{m-1}>3^{m-4} \cdot 2^{7} \\
>5 \cdot 3^{m-3} \cdot 2^{3}>3^{m-6} \cdot 2^{10}>3^{m-1} \cdot 2^{2}+1\end{array}$ \\
\hline$\vdots$ & $\vdots$ & $\vdots$ & $\vdots \quad$ (continued) \\
\hline
\end{tabular}


Table 2.5 Optimal Solution for IPP

\begin{tabular}{|c|c|c|c|}
\hline $\begin{array}{c}\text { given } \\
\text { quantity } \\
n\end{array}$ & $\begin{array}{c}\text { minimum } \\
\text { value } \\
g(n) \\
\end{array}$ & $\begin{array}{l}\text { optimal } \\
\text { policy } \\
\hat{\sigma}(n)\end{array}$ & $\begin{array}{l}\text { optimal } \\
\text { partition } \\
\qquad \geq n(=n \text { or }>n)\end{array}$ \\
\hline $3^{m-1} \cdot 2^{2}+1$ & $3 m+2$ & $2,3,4,5$ & $\begin{array}{l}3^{m} \cdot 2>3^{m-2} \cdot 2^{4}>5 \cdot 3^{m-1}>3^{m-4} \cdot 2^{7} \\
>5 \cdot 3^{m-3} \cdot 2^{3}>3^{m-6} \cdot 2^{10}>3^{m-1} \cdot 2^{2}+1\end{array}$ \\
\hline $3^{m-1} \cdot 2^{2}+2$ & $3 m+2$ & $2,3,4,5$ & $\begin{array}{l}3^{m} \cdot 2>3^{m-2} \cdot 2^{4}>5 \cdot 3^{m-1}>3^{m-4} \cdot 2^{7} \\
>5 \cdot 3^{m-3} \cdot 2^{3}>3^{m-6} \cdot 2^{10}>3^{m-1} \cdot 2^{2}+2\end{array}$ \\
\hline$\vdots$ & $\vdots$ & $\vdots$ & $\vdots$ \\
\hline $3^{m-6} \cdot 2^{10}$ & $3 m+2$ & $2,3,4,5$ & $\begin{array}{l}3^{m} \cdot 2>3^{m-2} \cdot 2^{4}>5 \cdot 3^{m-1}>3^{m-4} \cdot 2^{7} \\
>5 \cdot 3^{m-3} \cdot 2^{3}>3^{m-6} \cdot 2^{10}=3^{m-6} \cdot 2^{10}\end{array}$ \\
\hline $3^{m-6} \cdot 2^{10}+1$ & $3 m+2$ & $2,3,4,5$ & $\begin{array}{l}3^{m} \cdot 2>3^{m-2} \cdot 2^{4}>5 \cdot 3^{m-1}>3^{m-4} \cdot 2^{7} \\
>5 \cdot 3^{m-3} \cdot 2^{3}>3^{m-6} \cdot 2^{10}+1\end{array}$ \\
\hline $3^{m-6} \cdot 2^{10}+2$ & $3 m+2$ & $2,3,4,5$ & $\begin{array}{l}3^{m} \cdot 2>3^{m-2} \cdot 2^{4}>5 \cdot 3^{m-1}>3^{m-4} \cdot 2^{7} \\
>5 \cdot 3^{m-3} \cdot 2^{3}>3^{m-6} \cdot 2^{10}+2\end{array}$ \\
\hline$\vdots$ & $\vdots$ & $\vdots$ & $\vdots$ \\
\hline $5 \cdot 3^{m-3} \cdot 2^{3}$ & $3 m+2$ & $2,3,4,5$ & $\begin{array}{l}3^{m} \cdot 2>3^{m-2} \cdot 2^{4}>5 \cdot 3^{m-1}>3^{m-4} \cdot 2^{7} \\
>5 \cdot 3^{m-3} \cdot 2^{3}=5 \cdot 3^{m-3} \cdot 2^{3}\end{array}$ \\
\hline $5 \cdot 3^{m-3} \cdot 2^{3}+1$ & $3 m+2$ & $2,3,4,5$ & $\begin{array}{l}3^{m} \cdot 2>3^{m-2} \cdot 2^{4}>5 \cdot 3^{m-1}>3^{m-4} \cdot 2^{7} \\
>5 \cdot 3^{m-3} \cdot 2^{3}+1\end{array}$ \\
\hline $5 \cdot 3^{m-3} \cdot 2^{3}+2$ & $3 m+2$ & $2,3,4,5$ & $\begin{array}{l}3^{m} \cdot 2>3^{m-2} \cdot 2^{4}>5 \cdot 3^{m-1}>3^{m-4} \cdot 2^{7} \\
>5 \cdot 3^{m-3} \cdot 2^{3}+2\end{array}$ \\
\hline$\vdots$ & $\vdots$ & $\vdots$ & $\vdots$ \\
\hline $3^{m-4} \cdot 2^{7}$ & $3 m+2$ & $2,3,4,5$ & $\begin{array}{l}3^{m} \cdot 2>3^{m-2} \cdot 2^{4}>5 \cdot 3^{m-1}>3^{m-4} \cdot 2^{7} \\
=3^{m-4} \cdot 2^{7}\end{array}$ \\
\hline $3^{m-4} \cdot 2^{7}+1$ & $3 m+2$ & $2,3,4,5$ & $3^{m} \cdot 2>3^{m-2} \cdot 2^{4}>5 \cdot 3^{m-1}>3^{m-4} \cdot 2^{7}+1$ \\
\hline $3^{m-4} \cdot 2^{7}+2$ & $3 m+2$ & $2,3,4,5$ & $3^{m} \cdot 2>3^{m-2} \cdot 2^{4}>5 \cdot 3^{m-1}>3^{m-4} \cdot 2^{7}+2$ \\
\hline$\vdots$ & $\vdots$ & $\vdots$ & 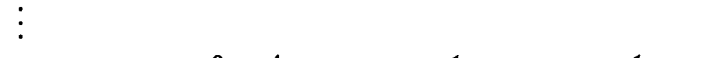 \\
\hline $5 \cdot 3^{m-1}$ & $3 m+2$ & $2,3,4,5$ & $3^{m} \cdot 2>3^{m-2} \cdot 2^{4}>5 \cdot 3^{m-1}=5 \cdot 3^{m-1}$ \\
\hline $5 \cdot 3^{m-1}+1$ & $3 m+2$ & $2,3,4$ & $3^{m} \cdot 2>3^{m-2} \cdot 2^{4}>5 \cdot 3^{m-1}+1$ \\
\hline $5 \cdot 3^{m-1}+2$ & $3 m+2$ & $2,3,4$ & $3^{m} \cdot 2>3^{m-2} \cdot 2^{4}>5 \cdot 3^{m-1}+2$ \\
\hline$\vdots$ & $\vdots$ & $\vdots$ & $\vdots$ \\
\hline $3^{m-2} \cdot 2^{4}$ & $3 m+2$ & $2,3,4$ & $3^{m} \cdot 2>3^{m-2} \cdot 2^{4}=3^{m-2} \cdot 2^{4}$ \\
\hline $3^{m-2} \cdot 2^{4}+1$ & $3 m+2$ & 2,3 & $3^{m} \cdot 2>3^{m-2} \cdot 2^{4}+1$ \\
\hline $3^{m-2} \cdot 2^{4}+2$ & $3 m+2$ & 2,3 & $3^{m} \cdot 2>3^{m-2} \cdot 2^{4}+2$ \\
\hline$\vdots$ & $\vdots$ & $\vdots$ & $\vdots$ \\
\hline $3^{m} \cdot 2$ & $3 m+2$ & 2,3 & $3^{m} \cdot 2=3^{m} \cdot 2$ \\
\hline $3^{m} \cdot 2+1$ & $\vdots$ & $\vdots$ & (continued) \\
\hline
\end{tabular}


Table 2.6 Optimal Solution for IPP

\begin{tabular}{|c|c|c|c|}
\hline $\begin{array}{c}\text { given } \\
\text { quantity } \\
n\end{array}$ & $\begin{array}{c}\text { minimum } \\
\text { value } \\
g(n) \\
\end{array}$ & $\begin{array}{l}\text { optimal } \\
\text { policy } \\
\hat{\sigma}(n) \\
\end{array}$ & $\begin{array}{l}\text { optimal } \\
\text { partition } \\
\qquad \geq n(=n \text { or }>n)\end{array}$ \\
\hline $\begin{array}{c}\vdots \\
3^{m} \cdot 2 \\
\end{array}$ & $\begin{array}{c}\vdots \\
3 m+2\end{array}$ & $\begin{array}{l}\vdots \\
2,3\end{array}$ & $\begin{array}{l}\vdots \\
3^{m} \cdot 2=3^{m} \cdot 2\end{array}$ \\
\hline $\begin{array}{l}3^{m} \cdot 2+1 \\
3^{m} \cdot 2+2\end{array}$ & $3 m+3$ & $2,3,4,5$ & $\begin{array}{l}3^{m+1}>3^{m-1} \cdot 2^{3}>3^{m-3} \cdot 2^{6} \\
>5 \cdot 3^{m-2} \cdot 2^{2}>3^{m-5} \cdot 2^{9}>3^{m} \cdot 2+1 \\
3^{m+1}>3^{m-1} \cdot 2^{3}>3^{m-3} \cdot 2^{6} \\
>5 \cdot 3^{m-2} \cdot 2^{2}>3^{m-5} \cdot 2^{9}>3^{m} \cdot 2+2\end{array}$ \\
\hline$\vdots$ & $\vdots$ & $\vdots$ & $\vdots$ \\
\hline $3^{m-5} \cdot 2^{9}$ & $3 m+3$ & $2,3,4,5$ & $\begin{array}{l}3^{m+1}>3^{m-1} \cdot 2^{3}>3^{m-3} \cdot 2^{6} \\
>5 \cdot 3^{m-2} \cdot 2^{2}>3^{m-5} \cdot 2^{9}=3^{m-5} \cdot 2^{9}\end{array}$ \\
\hline $3^{m-5} \cdot 2^{9}+1$ & $3 m+3$ & $2,3,4$ & $\begin{array}{l}3^{m+1}>3^{m-1} \cdot 2^{3}>3^{m-3} \cdot 2^{6} \\
>5 \cdot 3^{m-2} \cdot 2^{2}>3^{m-5} \cdot 2^{9}+1\end{array}$ \\
\hline $3^{m-5} \cdot 2^{9}+2$ & $3 m+3$ & $2,3,4$ & $\begin{array}{l}3^{m+1}>3^{m-1} \cdot 2^{3}>3^{m-3} \cdot 2^{6} \\
>5 \cdot 3^{m-2} \cdot 2^{2}>3^{m-5} \cdot 2^{9}+2\end{array}$ \\
\hline$\vdots$ & $\vdots$ & $\vdots$ & $\vdots$ \\
\hline $5 \cdot 3^{m-2} \cdot 2^{2}$ & $3 m+3$ & $2,3,4$ & $\begin{array}{l}3^{m+1}>3^{m-1} \cdot 2^{3}>3^{m-3} \cdot 2^{6} \\
5 \cdot 3^{m-2} \cdot 2^{2}=5 \cdot 3^{m-2} \cdot 2^{2}\end{array}$ \\
\hline $5 \cdot 3^{m-2} \cdot 2^{2}+1$ & $3 m+3$ & $2,3,4$ & $\begin{array}{l}3^{m+1}>3^{m-1} \cdot 2^{3}>3^{m-3} \cdot 2^{6} \\
>5 \cdot 3^{m-2} \cdot 2^{2}+1\end{array}$ \\
\hline $5 \cdot 3^{m-2} \cdot 2^{2}+2$ & $3 m+3$ & $2,3,4$ & $\begin{array}{l}3^{m+1}>3^{m-1} \cdot 2^{3}>3^{m-3} \cdot 2^{6} \\
>5 \cdot 3^{m-2} \cdot 2^{2}+2\end{array}$ \\
\hline$\vdots$ & $\vdots$ & $\vdots$ & $\vdots$ \\
\hline $3^{m-3} \cdot 2^{6}$ & $3 m+3$ & $2,3,4$ & $\begin{array}{l}3^{m+1}>3^{m-1} \cdot 2^{3}>3^{m-3} \cdot 2^{6} \\
=3^{m-3} \cdot 2^{6}\end{array}$ \\
\hline $3^{m-3} \cdot 2^{6}+1$ & $3 m+3$ & $2,3,4$ & $3^{m+1}>3^{m-1} \cdot 2^{3}>3^{m-3} \cdot 2^{6}+1$ \\
\hline $\begin{array}{c}3^{m-3} \cdot 2^{6}+2 \\
:\end{array}$ & $\begin{array}{c}3 m+3 \\
\quad:\end{array}$ & $\begin{array}{l}2,3,4 \\
:\end{array}$ & $\begin{array}{l}3^{m+1}>3^{m-1} \cdot 2^{3}>3^{m-3} \cdot 2^{6}+2 \\
:\end{array}$ \\
\hline $3^{m-1} \cdot 2^{3}$ & $3 m+3$ & $2,3,4$ & $3^{m+1}>3^{m-1} \cdot 2^{3}=3^{m-1} \cdot 2^{3}$ \\
\hline $3^{m-1} \cdot 2^{3}+1$ & $3 m+3$ & 3 & $3^{m+1}>3^{m-1} \cdot 2^{3}+1$ \\
\hline $3^{m-1} \cdot 2^{3}+2$ & $3 m+3$ & 3 & $3^{m+1}>3^{m-1} \cdot 2^{3}+2$ \\
\hline$\vdots$ & : & $\vdots$ & $\vdots$ \\
\hline $3^{m+1}$ & $3 m+3$ & 3 & $3^{m+1}=3^{m+1}$ \\
\hline$\vdots$ & $\vdots$ & $\vdots$ & $\vdots$ \\
\hline
\end{tabular}


Table 3.1 Upper-inverse Solution for MPP

\begin{tabular}{|c|c|c|c|}
\hline $\begin{array}{c}\text { given } \\
\text { quantity } \\
n\end{array}$ & $\begin{array}{c}\text { u-inverse } \\
\text { value } \\
f^{-1}(n)\end{array}$ & $\begin{array}{l}\text { composite } \\
\text { policy } \\
\pi^{*}\left(f^{-1}(n)\right)\end{array}$ & $\begin{array}{l}\text { resulting } \\
\text { partition } \\
n= \\
\end{array}$ \\
\hline 1 & 1 & - & - \\
\hline 2 & 2 & - & - \\
\hline 3 & 3 & - & - \\
\hline 4 & 4 & - & - \\
\hline 5 & 5 & 2,3 & $5=3+2$ \\
\hline 6 & 5 & 2,3 & $5=3+2$ \\
\hline 7 & 6 & 3 & $6=3+3$ \\
\hline 8 & 6 & 3 & $6=3+3$ \\
\hline 9 & 6 & 3 & $6=3+3$ \\
\hline 10 & 7 & $2,3,4$ & $7=3+2+2=3+4$ \\
\hline 11 & 7 & $2,3,4$ & $7=3+2+2=3+4$ \\
\hline 12 & 7 & $2,3,4$ & $7=3+2+2=3+4$ \\
\hline 13 & 8 & 2,3 & $8=3+3+2$ \\
\hline 14 & 8 & 2,3 & $8=3+3+2$ \\
\hline$\vdots$ & $\vdots$ & $\vdots$ & $\vdots$ \\
\hline 18 & 8 & 2,3 & $8=3+3+2$ \\
\hline 19 & 9 & 3 & $9=3+3+3$ \\
\hline 20 & 9 & 3 & $9=3+3+3$ \\
\hline$\vdots$ & $\vdots$ & $\vdots$ & $\vdots$ \\
\hline 27 & 9 & 3 & $9=3+3+3$ \\
\hline 28 & 10 & $2,3,4$ & $10=3+3+2+2=3+3+4$ \\
\hline 29 & 10 & $2,3,4$ & $10=3+3+2+2=3+3+4$ \\
\hline$\vdots$ & $\vdots$ & $\vdots$ & $\vdots$ \\
\hline 36 & 10 & $2,3,4$ & $10=3+3+2+2=3+3+4$ \\
\hline 37 & 11 & 2,3 & $11=3+3+3+2$ \\
\hline 38 & 11 & 2,3 & $11=3+3+3+2$ \\
\hline$\vdots$ & $\vdots$ & $\vdots$ & $\vdots$ \\
\hline 54 & 11 & 2,3 & $11=3+3+3+2$ \\
\hline 55 & 12 & 3 & $12=3+3+3+3$ \\
\hline 56 & 12 & 3 & $12=3+3+3+3$ \\
\hline$\vdots$ & $\vdots$ & $\vdots$ & $\vdots$ \\
\hline 81 & 12 & 3 & $12=3+3+3+3$ \\
\hline$\vdots$ & $\vdots$ & $\vdots$ & $\vdots$ \\
\hline
\end{tabular}


Table 3.2 Upper-inverse Solution for MPP

\begin{tabular}{|c|c|c|c|}
\hline $\begin{array}{c}\text { given } \\
\text { quantity } \\
n \\
\end{array}$ & $\begin{array}{c}\text { u-inverse } \\
\text { value } \\
f^{-1}(n) \\
\end{array}$ & $\begin{array}{l}\text { composite } \\
\text { policy } \\
\pi^{*}\left(f^{-1}(n)\right)\end{array}$ & $\begin{array}{l}\text { resulting } \\
\text { partition } \\
n=\end{array}$ \\
\hline$\vdots$ & $\vdots$ & $\vdots$ & $\vdots$ \\
\hline 54 & 11 & 2,3 & $11=3+3+3+2$ \\
\hline 55 & 12 & 3 & $12=3+3+3+3$ \\
\hline 56 & 12 & 3 & $12=3+3+3+3$ \\
\hline$\vdots$ & $\vdots$ & $\vdots$ & $\vdots$ \\
\hline 81 & 12 & 3 & $12=3+3+3+3$ \\
\hline$\vdots$ & $\vdots$ & $\vdots$ & $\vdots$ \\
\hline $2 \cdot 3^{m-1}+1$ & $3 m$ & 3 & $3 m=3+\cdots 3$ \\
\hline $2 \cdot 3^{m-1}+2$ & $3 m$ & 3 & $3 m=3+\cdots 3$ \\
\hline$\vdots$ & $\vdots$ & $\vdots$ & (m-times) \\
\hline $3^{m}$ & $3 m$ & 3 & $3 m=3+\cdots 3$ \\
\hline $3^{m}+1$ & $3 m+1$ & $2,3,4$ & $3 m+1=3+\cdots 3+2+2=3+\cdots 3+4$ \\
\hline $3^{m}+2$ & $3 m+1$ & $2,3,4$ & $3 m+1=3+\cdots 3+2+2=3+\cdots 3+4$ \\
\hline$\vdots$ & $\vdots$ & $\vdots$ & $(m-1$-times $)$ \\
\hline $4 \cdot 3^{m-1}$ & $3 m+1$ & $2,3,4$ & $3 m+1=3+\cdots 3+2+2=3+\cdots 3+4$ \\
\hline $4 \cdot 3^{m-1}+1$ & $3 m+2$ & 2,3 & $3 m+2=3+\cdots 3+2$ \\
\hline $4 \cdot 3^{m-1}+2$ & $3 m+2$ & 2,3 & $3 m+2=3+\cdots 3+2$ \\
\hline$\vdots$ & $\vdots$ & $\vdots$ & $(m$-times $)$ \\
\hline $2 \cdot 3^{m}$ & $3 m+2$ & 2,3 & $3 m+2=3+\cdots 3+2$ \\
\hline$\vdots$ & $\vdots$ & $\vdots$ & $\vdots$ \\
\hline
\end{tabular}


Table 4 Lower-inverse Solution for IPP

\begin{tabular}{|c|c|c|c|}
\hline $\begin{array}{c}\text { given } \\
\text { quantity } \\
n\end{array}$ & $\begin{array}{c}\text { l-inverse } \\
\text { value } \\
g_{-1}(n)\end{array}$ & $\begin{array}{l}\text { composite } \\
\text { policy } \\
\hat{\sigma}\left(g_{-1}(n)\right)\end{array}$ & $\begin{array}{l}\text { resulting } \\
\text { partition } \\
\qquad n(=n \text { or }>n)\end{array}$ \\
\hline 1 & 1 & - & - \\
\hline 2 & 2 & - & - \\
\hline 3 & 3 & - & - \\
\hline 4 & 4 & - & - \\
\hline 5 & 6 & 2,3 & - \\
\hline 6 & 9 & 3 & $3 \cdot 3=9$ \\
\hline 7 & 12 & $2,3,4$ & $3 \cdot 2 \cdot 2=3 \cdot 4=12$ \\
\hline 8 & 18 & 2,3 & $3 \cdot 3 \cdot 2=18$ \\
\hline 9 & 27 & 3 & $3 \cdot 3 \cdot 3=27$ \\
\hline 10 & 36 & $2,3,4$ & $3 \cdot 3 \cdot 2 \cdot 2=3 \cdot 3 \cdot 4=36$ \\
\hline 11 & 54 & 2,3 & $3 \cdot 3 \cdot 3 \cdot 2=54$ \\
\hline 12 & 81 & 3 & $3 \cdot 3 \cdot 3 \cdot 3=81$ \\
\hline 13 & 108 & $2,3,4$ & $3 \cdot 3 \cdot 3 \cdot 2 \cdot 2=3 \cdot 3 \cdot 3 \cdot 4=108$ \\
\hline 14 & 162 & 2,3 & $3 \cdot 3 \cdot 3 \cdot 3 \cdot 2=162$ \\
\hline 15 & 243 & 3 & $3 \cdot 3 \cdot 3 \cdot 3=243$ \\
\hline 16 & 324 & $2,3,4$ & $3 \cdot 3 \cdot 3 \cdot 3 \cdot 2 \cdot 2=3 \cdot 3 \cdot 3 \cdot 3 \cdot 4=324$ \\
\hline 17 & 486 & 2,3 & $3 \cdot 3 \cdot 3 \cdot 3 \cdot 3 \cdot 2=486$ \\
\hline$\vdots$ & $\vdots$ & $\vdots$ & $\vdots$ \\
\hline $3 m$ & $3^{m}$ & 3 & $3 \cdot 3 \cdots 3=3^{m}$ \\
\hline $3 m+1$ & $4 \cdot 3^{m-1}$ & $2,3,4$ & $3 \cdot 3 \cdots 3 \cdot 2 \cdot 2=3 \cdot 3 \cdots 3 \cdot 4=4 \cdot 3^{m-1}$ \\
\hline $3 m+2$ & $2 \cdot 3^{m}$ & 2,3 & $3 \cdot 3 \cdots 3 \cdot 2=2 \cdot 3^{m}$ \\
\hline$\vdots$ & $\vdots$ & $\cdot$ & $\vdots$ \\
\hline
\end{tabular}

\section{Acknowledgments}

The authors would like to thank the anonymous referee for careful reading of this paper and helpful comments.

\section{References}

Beckmann, M.J. and Laderman, J. (1956), A bound on the use of inefficient indivisible units, Naval Research Logistics Quartely, 3:245-252.

Beckmann, M.J. (1968), Dynamic Programming of Economic Decisions, Springer, NY. Bellman, R.E. (1957), Dynamic Programming, Princeton Univ. Press, NJ.

Ibaraki, T. and Katoh, N. (1988), Resource Allocation Problems: Algorithmic Approaches, MIT Press, MA.

Golmb, S.W. (1968), E2118, Amer. Math. Monthly, 75:878. 
Golmb, S.W. (1980), Iterated binomial coefficients, Amer. Math. Monthly, 87:719-727. Iwamoto, S. (1977a), Inverse theorem in dynamic programming I, J. Math. Anal. Appl., 58:113-113.

Iwamoto, S. (1977b), Inverse theorem in dynamic programming II, J. Math. Anal. Appl., 58:249-279.

Iwamoto, S. (1977c), Inverse theorem in dynamic programming III, J. Math. Anal. Appl., 58:439-448.

Iwamoto, S. (1983), A dynamic inversion of the classical variational problems, J. Math. Anal. Appl., 100:354-374.

Iwamoto, S. and Wang, C.-L. (1983), Continuous dynamic programming approach to inequalities, J. Math. Anal. Appl., 96:119-129.

Iwamoto, S. and Wang, C.-L. (1986), Continuous dynamic programming approach to inequalities II, J. Math. Anal. Appl., 118:279-286.

Iwamoto, S. (1987), Theory of Dynamic Programs, (in Japanese), Kyushu Univ. Press, Fukuoka.

Sniedovich, M. (1992), Dynamic Programming, Marcel Dekker, Inc. NY.

Received October 8, 1998 